\title{
Eine kontinuierliche Arbeitskräfteerhebung für die Europäische Union *
}

\author{
Aloïs Van Bastelaer, Eurostat
}

\begin{abstract}
Zusammenfassung: Statistiken zu Arbeitsmarkt und Beschäftigung sind im Hinblick auf mittelfristige Programme wie NAP, Agenda 2000 usw. von ebenso großer wie offenkundiger Bedeutung. Die jährliche ArbeitskräfteErhebung (AKE) ist in Österreich mit der EU-Mitgliedschaft 1995 obligatorisch geworden, sie ist aber immer wieder inhaltlichen, z.T. auch methodischen Änderungen unterworfen. Durch Ratsverordnung (VO) vorgeschrieben, müssen die Erhebungen von den nationalen statistischen Ämtern durchgeführt werden. 1998 liegt dem Rat abermals eine neue Fassung der VO vor, die voraussichtlich für Erhebungen ab dem Berichtsjahr 1998 Geltung haben wird. Diese - gravierende - Novellierung betrifft neben einigen inhaltlichen Revisionen v.a. die Einführung einer „kontinuierlichen“ Erhebung. Für Mitgliedstaaten, denen diese Änderung aus organisatorischen Gründen nicht (sofort) möglich ist, besteht aber weiterhin die Option, bei der bisherigen einmaligen Erhebung pro Jahr zu bleiben (dazu zählt auch Österreich).
\end{abstract}

\begin{abstract}
With regard to medium term projects like NAP, Agenda 2000 ... labour market and employment statistics are of great and evident interest. In Austria the yearly Labour Force Survey (LFS) became obligatory with the EU membership in 1995 but, nevertheless, changes of contents and methods happen quite often. The national statistical offices have to organize the surveys according to the respective Council Regulation. In 1998 a new version of this Regulation will be issued, to be valid from 1998 onwards. Apart from some revisions of the contents, this important amendment mainly concerns the introduction of a "continuous survey". For member countries not able to change to a continuous survey immediately, the possibility to leave the LFS unchanged (once a year survey) is provided, for the time being.
\end{abstract}

\section{Hintergrund}

Ab 1999 wird in der Europäischen Union (mit Ausnahme Deutschlands und Österreichs) eine kontinuierliche Arbeitskräfteerhebung, die vierteljährliche Ergebnisse liefert, durchgeführt werden. Das Design dieser kontinuierlichen Arbeitskräfteerhebung

* Schriftliche ausführliche Ausarbeitung eines Vortrages, gehalten an den „Österreichischen Statistik-Tagen 1997“ der Österreichischen Statistischen Gesellschaft in Wien. 
wurde von der Arbeitsgruppe Beschäftigungsstatistik 1996 und 1997 erörtert. Am 12. November 1997 wurde die neue Verordnung für diese Erhebung dem Rat vorgelegt.

Die kontinuierliche Arbeitskräfteerhebung tritt an die Stelle der derzeitigen jährlichen Arbeitskräfteerhebung der Gemeinschaft, die seit 1983 jeweils im Frühjahr durchgeführt wird. Die Veränderungen des Designs der Arbeitskräfteerhebung sind erheblich, weil sich der Übergang von einer jährlichen Erhebung hin zu einer kontinuierlichen Erhebung auf die Feldarbeit, die Aufbereitung und die Freigabe der Daten auswirkt. Änderungen sind notwendig, weil

- neuer Bedarf seitens der Nutzer berücksichtigt werden muß, und

- ein Erhebungsdesign erforderlich ist, das gewährleistet, daß auf Gemeinschaftsebene vergleichbare Daten über den Arbeitsmarkt bereitgestellt werden; dieses Erhebungsdesign soll als Modell für die Entwicklung einer Arbeitskräfteerhebung in den Ländern dienen, die möglicherweise später der Europäischen Union beitreten.

Diese Forderung vergleichbarer Daten ist ausschlaggebend für die wesentlichen Erhebungscharakteristika der kontinuierlichen Arbeitskräfteerhebung. Es sollte vermieden werden, daß die Interpretation der Daten durch Unterschiede zwischen den nationalen Erhebungsdesigns beeinflußt wird. Diese Erhebungscharakteristika sind in der neuen Ratsverordnung festgelegt und werden in den nachfolgenden Abschnitten erörtert. Sie beziehen sich auf

- die zu erfassende Grundgesamtheit und die Erhebungseinheiten,

- die erforderliche statistische Genauigkeit,

- die Häufigkeit der Erhebung und den Bezugszeitraum; obwohl die Durchführung der Feldarbeit nicht von der Ratsverordnung erfaßt wird, werde ich sie im Zusammenhang mit dem Bezugszeitraum in einer kontinuierlichen Erhebung erörtern,

- das Gewichtungsverfahren,

- die Variablen,

- das Programm von Ad-hoc-Modulen zu spezifischen Bereichen,

- die Durchführung der Erhebung und

- die Frist für die Übermittlung der Ergebnisse.

\section{Ziele der kontinuierlichen Arbeitskräfteerhebung}

Mit der kontinuierlichen Arbeitskräfteerhebung werden folgende Ziele angestrebt:

i) vierteljährliche Trendschätzungen von Beschäftigung und Arbeitslosigkeit,

ii) Strukturdaten zu Beschäftigung und Arbeitslosigkeit, insbesondere auf regionaler Ebene, 
iii) Schätzung des Volumens der Beschäftigung und der Unterbeschäftigung,

iv) Daten über Haushalte.

ad i) Beschäftigung und Arbeitslosigkeit sind politische Kernbereiche innerhalb der EU. Die Festlegung einer Arbeitsmarktpolitik ist abhängig von der Verfügbarkeit international vergleichbarer Daten und der Entwicklung geeigneter Instrumente zur Überwachung von Beschäftigungstrends (Europäischer Rat Dublin). Diese Beschäftigungstrends können mit wirtschaftlicher Erholung oder Rezession in Zusammenhang gebracht werden. Es werden kurzfristige Indikatoren des Arbeitsangebots benötigt, untergliedert nach Geschlecht, Alter, Wirtschaftszweig, Beruf, Art des Arbeitsvertrags und Bildungsabschluß.

ad ii) Neben den kurzfristigen Indikatoren werden umfassende und erschöpfende Angaben zur Struktur der Erwerbsbevölkerung in den EU-Regionen benötigt. Die Angaben sind umfassend und erschöpfend, weil die Arbeitskräfteerhebung erwerbsstatistische Merkmale (für eine Stichprobe) der gesamten Wohnbevölkerung bereitstellt. Es handelt sich um Angaben zur durchschnittlichen Beschäftigung und Arbeitslosigkeit für ein ganzes Jahr. Dabei sollte das saisonale Muster der Beschäftigung und nicht nur die spezielle Situation auf dem Arbeitsmarkt während einer bestimmten Woche oder eines bestimmten Monats des Jahres abgebildet werden.

ad iii) Zusätzlich zur Erfassung der Anzahl der erwerbstätigen Personen besteht Bedarf an Schätzungen des Arbeitsvolumens. In der Vergangenheit gingen fast alle Beschäftigten einer Vollzeittätigkeit nach, doch eine Beschreibung des heutigen Arbeitsmarkts allein mit Blick auf die Zahl der beschäftigten Personen ist unzureichend, denn $16 \%$ aller Erwerbstätigen in der EU haben einen Teilzeitarbeitsplatz. Hinzu kommt, daß Teilzeitarbeit unterschiedliche Stundenzahlen umfaßt. Rund $20 \%$ der Teilzeitbeschäftigten in der EU arbeiten in der Regel weniger als 11 Stunden pro Woche, 10\% arbeiten mehr als 30 Stunden. Eine vollständige Beschreibung des Arbeitsmarktes erfordert Volumenschätzungen, die das Ausmaß an Teilzeitarbeit berücksichtigen. Bei einer verläßlichen Volumenschätzung muß auch die saisonale Struktur der Beschäftigung berücksichtigt werden.

ad iv) Des weiteren ist die Arbeitskräfteerhebung eine wichtige Quelle für Daten über Haushalte. Die Daten über den Erwerbsstatus der einzelnen Haushaltsmitglieder ermöglichen eine kontextbezogene Analyse der Erwerbsbeteiligung der einzelnen Haushaltsmitglieder. Als umfangreiche Erhebung ist die Arbeitskräfteerhebung ebenfalls eine einzigartige Quelle für genaue Informationen über die Zusammensetzung von Haushalten und kann zur Aktualisierung der Volkszählungsdaten herangezogen werden. 


\section{Zu erfassende Grundgesamtheit und Erhebungs- einheiten}

Bei der Definition der Grundgesamtheit in der Arbeitskräfteerhebung wird das Kriterium des ,üblichen Wohnsitzes“ verwendet (Inländerkonzept). Die durch die Arbeitskräfteerhebungen in der EU erfaßte Grundgesamtheit besteht aus allen Einwohnern im Alter von 15 Jahren und darüber, mit Ausnahme der Angehörigen ausländischer diplomatischer Vertretungen und ausländischer Streitkräfte. Diese Grundgesamtheit umfaßt alle Personen, die in privaten Haushalten leben. Sie wird in Abhängigkeit von der jeweiligen Erhebungspraxis ergänzt durch Erhebungen bei Personen, die in Anstaltshaushalten leben. Einige Kategorien von Anstaltshaushalten, beispielsweise Arbeiter, die in Heimen leben, dürfen nicht von der Erhebung ausgenommen werden. In Belgien, Dänemark, Finnland und Schweden werden alle in Anstaltshaushalten lebenden Personen vollständig erfaßt, weil als Auswahlgrundlage das Bevölkerungsregister herangezogen wird und die in Anstaltshaushalten lebenden Personen nicht gesondert aufgeführt werden. Die Anstaltshaushalte können auch durch eine gesonderte Stichprobe erfaßt werden (im Vereinigten Königreich beispielsweise besteht eine gesonderte Stichprobe für Krankenhäuser und Pflegeheime), oder es können Proxydaten bei der Adresse der Familie/bei dem Haushalt gesammelt werden, mit dem die betreffenden Personen weiterhin in Beziehung stehen (beispielsweise Internatsschüler). Ein Vergleich der Erfassung bestimmter Kategorien von in Anstaltshaushalten lebenden Personen durch die Arbeitskräfteerhebung mit anderen Quellen kann bei der Entscheidung helfen, ob die Stichprobe angemessen ist oder erweitert werden muß.

\section{Anforderungen an die statistische Genauigkeit}

In bezug auf das erste und das zweite Ziel (vierteljährliche Trendschätzungen und Strukturdaten zu Beschäftigung und Arbeitslosigkeit auf regionaler Ebene) werden zwei statistische Genauigkeitsanforderungen festgelegt. Die Genauigkeitsanforderungen werden ausgedrückt als relative Standardfehler von Merkmalen, die sich auf $5 \%$ der Bevölkerung im erwerbsfähigen Alter beziehen.

Die erste Anforderung betrifft Jahresdurchschnitte auf regionaler Ebene: Der relative Standardfehler der jährlichen Durchschnitte auf Ebene Nuts II (in Österreich: Bundesländer) darf nicht höher als $8 \%$ liegen. Diese Anforderung entspricht derjenigen in der derzeit geltenden Verordnung über die jährliche Arbeitskräfteerhebung in der Gemeinschaft. Beim geschätzten Standardfehler sollte das Stichprobendesign (Schichtung und Klumpenbildung) sowie der Schätzer (Stichprobenwahrscheinlichkeiten und Ex-post-Stratifizierung) berücksichtigt werden. Da dieser Designeffekt abhängig ist von der betrachteten Variablen, sollte aus praktischen Gründen der Designeffekt für die Schätzung der Arbeitslosigkeit berücksichtigt werden.

Die zweite Anforderung bezieht sich auf die absolute Größe der vierteljährlichen Nettoveränderungen: Der relative Standardfehler vierteljährlicher Veränderungen auf nationaler Ebene darf nicht über $2 \%$ liegen; für Mitgliedstaaten mit einer Bevölkerung 
zwischen 1 und 20 Millionen Einwohnern wird diese Anforderung gelockert, d. h. der Standardfehler vierteljährlicher Veränderungen darf nicht über 3 \% liegen.

\section{Häufigkeit der Erhebung und Bezugszeitraum}

\subsection{Eine kontinuierliche Erhebung}

Der Hauptgrund für eine kontinuierliche Erhebung liegt im statistischen Bereich. Im Hinblick auf die Ziele (ii) (Strukturdaten zur jahresdurchschnittlichen Beschäftigung und Arbeitslosigkeit) und (iii) (Volumenschätzung von Beschäftigung und Arbeitslosigkeit) sollten die Bezugswochen gleichmäßig über das gesamte Jahr verteilt werden. Die Schwankungen von Beschäftigung und Arbeitslosigkeit in einem Quartal in einer Beschreibung des Arbeitsmarktes müssen berücksichtigt werden. Dieser Definition steht die Definition des Erhebungsbezugszeitraums in Form einer einzelnen Woche gegenüber. Das Berichtswochenkonzept ist von der Volkszählung inspiriert. Ziel der Volkszählung ist die Zählung - und Aufgliederung - der gesamten Wohnbevölkerung an einem einzigen Bezugstag. Wenn es um einen Stichtag geht, spielen bei den hier zu diskutierenden Sachverhalten Veränderungen innerhalb der Bezugsperiode naturgemäß keine Rolle. Statistiken auf der Grundlage einer einzigen Bezugswoche sind über einen längeren Zeitraum nicht repräsentativ für die Erwerbsbevölkerung.

Die kontinuierliche Arbeitskräfteerhebung der Gemeinschaft wird vierteljährliche und jährliche Ergebnisse (Ziele i und ii) bereitstellen. Für jedes Quartal wird eine repräsentative Stichprobe benötigt. Die Anzahl der repräsentativen Stichproben bestimmt die Häufigkeit einer Erhebung (Tabelle 1). Die Erhebung in Schweden beispielsweise besteht aus monatlichen Stichproben. Der Mikrozensus in Österreich besteht aus vierteljährlichen Stichproben und die Arbeitskräfteerhebung im Vereinigten Königreich aus Stichproben über 13 Wochen; jede Stichprobe von 13 aufeinanderfolgenden Wochen ist für das Vereinigte Königreich repräsentativ. Die Erhebung in Frankreich besteht aus einer jährlichen Stichprobe.

Tabelle 1: Häufigkeit und Bezugszeitraum der Arbeitskräfteerhebung 1997

\begin{tabular}{|c|c|c|c|c|c|}
\hline monatlich & & vierteljährlich & & jährlich & \\
\hline kontinuierlich & eine Woche & kontinuierlich & $\begin{array}{l}\text { eine oder mehrere } \\
\text { Wochen }\end{array}$ & April, Mai, Juni & eine Woche \\
\hline Niederlande & $\begin{array}{l}\text { Finnland } \\
\text { (Woche vom } \\
\text { 15.) }\end{array}$ & Dänemark & $\begin{array}{l}\text { Italien (erste volle } \\
\text { Woche des ersten } \\
\text { Monats in jedem } \\
\text { Quartal) }\end{array}$ & Frankreich & Deutschland \\
\hline Spanien & & $\begin{array}{l}\text { Vereinigtes } \\
\text { Königreich }\end{array}$ & $\begin{array}{l}\text { Österreich (die } \\
\text { ersten } 3 \text { Wochen } \\
\text { des letzten Monats } \\
\text { in jedem Quartal) }\end{array}$ & Griechenland & Belgien \\
\hline Schweden & & & Portugal & Irland & Luxemburg \\
\hline
\end{tabular}


Das neue Design der Arbeitskräfteerhebung in Frankreich, Griechenland, Irland, Belgien und Luxemburg beinhaltet eine Änderung der Häufigkeit der Erhebung und eine Neuverteilung der Bezugswochen innerhalb eines Monats oder eines Quartals. In Finnland, Italien und Portugal wird das neue Design der Erhebung zu einer Neuverteilung der Bezugswochen führen.

\subsection{Erhebung vom Typ "Rotierendes Panel"}

Die Schätzungen aus der kontinuierlichen Arbeitskräfteerhebung der Gemeinschaft müssen zwei Anforderungen im Hinblick auf die statistische Genauigkeit erfüllen: Ein vorgegebener relativer Standardfehler für jährliche Durchschnitte auf regionaler Ebene und einer für vierteljährliche Nettoveränderungen darf nicht überschritten werden. Eine Erhebung mit rotierendem Panel ist eine effiziente Methode zur Messung vierteljährlicher Veränderungen. Verglichen mit unabhängigen Stichproben erhöht die gemeinsame Stichprobe über zwei Quartale die Genauigkeit der Unterschiede zwischen zwei Niveauschätzungen unter der Voraussetzung, daß die Korrelation zwischen den Variablen zu den beiden Zeitpunkten positiv ist. Dieser Gewinn an Genauigkeit für Veränderungsschätzungen könnte durch die Mobilität der Auskunftspersonen verringert werden. Haushalte, die aus einer in der Stichprobe befindlichen Wohnung ausziehen, können durch einziehende Haushalte ersetzt werden, wenn keine Daten über individuelle Veränderungen verlangt werden. Diese unterschiedlichen Haushalte können die Korrelation im Laufe der Zeit verringern, und zwar in dem Ausmaß, in dem ihre Merkmale anders sind. Eine Panelerhebung mit einer vierteljährlichen Rotation hat völlig andere Auswirkungen auf Schätzungen jährlicher Ergebnisse. Ein Aggregat aus teilweise gemeinsamen vierteljährlichen Stichproben enthält wiederholte Beobachtungen zur selben Stichprobenperson, die gesamte Stichprobengröße ist also geringer als die Anzahl der Beobachtungen. Ein Satz monatlicher oder vierteljährlicher unabhängiger Stichproben ist für die zuletzt genannten Jahresschätzungen effizienter, wobei stets eine positive Korrelation im Zeitablauf zwischen den einzelnen Beobachtungen angenommen wird.

Eine Panelerhebung mit vierteljährlicher Rotation hat ein hohes Analysepotential. Es besteht offensichtlich Bedarf an Daten über Unsicherheit des Arbeitsplatzes sowie Zugänge in und Abgänge aus Beschäftigung. Indikatoren für unsichere Arbeitsplätze sind befristete Arbeitsverhältnisse oder Gelegenheitsjobs. Einsteiger und Wiedereinsteiger in den Arbeitsmarkt sowie Personen, die den Arbeitsplatz wechseln, haben unter Umständen verschiedene Möglichkeiten, eine Beschäftigung zu bekommen, und sie können Arbeitsplätze mit unterschiedlichen Arbeitsverträgen erhalten. Personen, die ihren Arbeitsplatz aus eigener Entscheidung aufgeben, scheiden entweder aus dem Erwerbsleben aus oder nehmen sofort eine neue Beschäftigung auf. Personen, die ihren Arbeitsplatz verlieren, werden hingegen arbeitslos und finden möglicherweise nach einiger Zeit, abhängig von der Nachfrage, ihren Fähigkeiten und Berufserfahrungen eine neue Stelle. Stehen in allen Quartalen für ein und dieselbe Person Beobachtungen zur Verfügung, können diese diskreten Daten miteinander verknüpft werden. Danach ist es möglich, die Grundgesamtheit über den Zeitraum der vergangenen 12 Monate hinsichtlich ihres Erwerbsstatus zu beschreiben. 
Die kontinuierliche Arbeitskräfteerhebung der Gemeinschaft beinhaltet Daten über Löhne und Gehälter. Daten über Löhne und Gehälter dienen dem besseren Verständnis des Arbeitsmarkts und der Arbeitsbedingungen. Sie beschreiben, welches Einkommen in einzelnen Berufen und Wirtschaftszweigen erzielt wird. Sie ermöglichen eine Analyse der Verteilung der Löhne und Gehälter nach Art des Arbeitsplatzes (Teilzeit oder Vollzeit, unbefristeter oder befristeter Arbeitsvertrag) sowie im Zusammenhang mit ansteigender oder sinkender Arbeitskräftenachfrage. Die zu sammelnden Daten über Löhne und Gehälter sind der monatliche Nettoverdienst und die jährliche Nettogesamtsumme zusätzlicher Zahlungen, die nicht monatlich erfolgen. Eine Frage nach dem monatlichen Nettoverdienst erfüllt die Bedingung einer minimalen Belastung durch die Befragung. Wenn die Daten in allen Quartalen gesammelt werden, kann der jährliche Nettoverdienst einer Person, die während des ganzen Jahres erwerbstätig war, als Durchschnitt der vierteljährlichen Summen plus der Gesamtsumme zusätzlicher Zahlungen errechnet werden.

Die endgültige Auswahl des Rotationsmusters ist abhängig von einem komplizierten Austarieren mehrerer Faktoren: Die Genauigkeit vierteljährlicher Veränderungsschätzungen und jährlicher Durchschnitte, die analytische Verwendung der Daten und die Panelsterblichkeit. Ein erster zu berücksichtigender Aspekt ist die Ineffizienz eines Aggregats aus teilweise gemeinsamen vierteljährlichen Stichproben für jährliche Gesamtschätzungen. Wird die Stichprobenüberlappung zwischen zwei Quartalen vergrößert, so muß der gesamte Stichprobenumfang erhöht werden, damit dieselbe Genauigkeit für jährliche Gesamtschätzungen erzielt werden kann wie vorher bei unabhängigen Stichproben. Zum zweiten verbessert ein hoher Überschneidungsgrad der Stichprobe zwischen Quartalen die Genauigkeit vierteljährlicher Veränderungsschätzungen und ermöglicht die Verknüpfung der diskreten Beobachtungen für Einzelpersonen über mehrere Quartale. Doch führen wiederholte Beobachtungen zu einer erhöhten Belastung und damit möglicherweise zu Panelsterblichkeit. Neben diesen Überlegungen im Zusammenhang mit der Analyse vierteljährlicher Veränderungen und jährlicher Gesamtschätzungen besteht möglicherweise Bedarf an einer Analyse jährlicher Trendschätzungen und langfristiger Veränderungen. Für diese Art der Analyse sind wiederholte Beobachtungen derselben Einzelpersonen im Abstand von einem Jahr erforderlich. Zusätzlich zu einer vierteljährlichen Rotation würde eine jährliche Rotation benötigt.

Es gibt eine Vielzahl von Rotationsmustern für die nationalen Arbeitskräfteerhebungen entsprechend den statistischen Prioritäten und den Bedingungen für die Datensammlung (Tabelle 2). Das Rotationsmuster wird von den Mitgliedstaaten unter Berücksichtigung der zwei statistischen Genauigkeitsanforderungen festgelegt.

Die Erhebungen, bei denen sich die vierteljährlichen Stichproben zu einem Drittel und mehr überschneiden, beinhalten ein Interview in mindestens drei Quartalen. Hauptziel der Rotationsmuster mit einem gemeinsamen vierteljährlichen Stichprobenumfang von mindestens drei Fünfteln ist die Genauigkeit vierteljährlicher Veränderungen. Insgesamt 8 Interviews in Österreich und Schweden sind relativ viel. Diese hohe Zahl wiederholter Beobachtungen bietet den Vorteil, daß ein Kerndatensatz von Arbeitsmarktvariablen für dieselbe Unterstichprobe 8 Quartale hindurch zur Verfügung steht und daß die Beziehungen zwischen diesem Kerndatensatz und einem ergänzenden, aber variablen Fragenblock analysiert werden kann. Die Bereiche, die diese Fragen 
ansprechen, werden in jedem Quartal verändert und zusätzlich zum Kernfragebogen der Arbeitskräfteerhebung gestellt (siehe Abschnitt 8).

Die Haushalte müssen nicht in jedem Quartal interviewt werden. Haushalte können nach einigen Interviews zeitweise aus dem Panel herausgenommen und dann zwei oder drei Quartale später wieder aufgenommen werden (Finnland, Italien und Dänemark). Diese Designs stellen einen Kompromiß zwischen den zwei statistischen Anforderungen dar: Genauigkeit vierteljährlicher Veränderungen und jährlicher Gesamtwerte. Die Genauigkeit der vierteljährlichen Veränderungen wird so durch gemeinsame Stichproben von Quartal zu Quartal - verglichen mit unabhängigen Stichproben verbessert, wobei ein geringerer Genauigkeitsverlust bei den jährliche Gesamtschätzwerten auftritt als bei einer Rotation in jeweils aufeinanderfolgenden Quartalen. In Frankreich, Deutschland und Griechenland verbleiben Haushalte drei oder vier Jahre lang in der Stichprobe, so daß langfristige Veränderungen analysiert werden können. Die Eurostat-Anforderung, ein erneutes Interview bei einem Viertel der Stichprobe im Folgejahr durchzuführen, gilt nicht mehr für die kontinuierliche Arbeitskräfteerhebung der Gemeinschaft. Das Design in Irland, Belgien, Luxemburg und in den Niederlanden entspricht der Eurostat-Anforderung, nur umfaßt in Belgien die gemeinsame Stichprobe $30 \%$ anstelle von $25 \%$.

Tabelle 2: Gemeinsame Stichprobengrößen von Jahr zu Jahr und von Quartal zu Quartal

\begin{tabular}{|c|c|c|c|c|c|c|c|}
\hline & Von Jahr zu & ahr: & & & & & \\
\hline $\begin{array}{l}\text { von } \\
\text { Quartal zu } \\
\text { Quartal }\end{array}$ & $1 / 5$ & $1 / 4$ & $1 / 3$ & $2 / 5$ & $1 / 2$ & $2 / 3$ & $3 / 4$ \\
\hline $\begin{array}{l}\text { keine ge- } \\
\text { meinsame } \\
\text { Stichprobe }\end{array}$ & & $\begin{array}{l}\text { Irland", } \\
\text { Luxem- } \\
\text { burg, } \\
\text { Nieder- } \\
\text { lande }\end{array}$ & Belgien & & & $\begin{array}{l}\text { Frank- } \\
\text { reich }\end{array}$ & $\begin{array}{l}\text { Deutsch- } \\
\text { land, } \\
\text { Griechen- } \\
\text { land }\end{array}$ \\
\hline $1 / 3$ & & & Dänemark & & & & \\
\hline $1 / 2$ & & & & & Italien & & \\
\hline $3 / 5$ & & & & Finnland & & & \\
\hline $4 / 5$ & $\begin{array}{l}\text { Vereinigtes } \\
\text { Königreich }\end{array}$ & & & & & & \\
\hline $5 / 6$ & & & $\begin{array}{l}\text { Spanien, } \\
\text { Portugal }\end{array}$ & & & & \\
\hline $7 / 8$ & & & & & $\begin{array}{l}\text { Schweden } \\
\text { Österreich }\end{array}$ & & \\
\hline
\end{tabular}


Eine in einer Panel-Erhebung zweimal gestellte Frage wird von der Auskunftsperson möglicherweise unterschiedlich interpretiert. Um zu vermeiden, daß eine Änderung eines Merkmals angegeben wird, das tatsächlich unverändert geblieben ist, werden dem Interviewer die Antworten an die Hand gegeben, die beim vorhergehenden Interview gegeben wurden. Diese Antworten werden der Auskunftsperson vorgetragen, und sie gibt an, ob sich die Situation geändert hat. Diese Art des Interviews ("dependent interviewing") kann für Merkmale verwendet werden, die sich auf die übliche Situation beziehen, oder dann, wenn keine Veränderung zu erwarten ist. Sobald feststeht, daß die Auskunftsperson einen Arbeitsplatz hat, kann gefragt werden, ob sie immer noch dem selben Beruf nachgeht und beim selben Arbeitgeber beschäftigt ist (Hill, 1994). Ein Interview mit Vorgaben trägt zu einem einheitlicheren Muster bei. Dabei wird auch die Rolle der Auskunftsperson anerkannt, in dem auf Informationen Bezug genommen wird, die beim letzten Interview (Statistics Sweden, 1989) geliefert wurden. Ein Test von Statistics Sweden im November 1984 bei einer Stichprobe von 4000 Personen zwei Monate nach dem ersten Interview zeigte, daß eine geringere Zahl von Veränderungen bei der Arbeitsplatzbindung 1 zu verzeichnen war, wenn ein Interview mit Vorgaben durchgeführt wurde $(3,5 \%$ gegenüber fast $5 \%$, wenn der Interviewer bei beiden Befragungen dieselbe Person war oder $8 \%$ im Falle unterschiedlicher Interviewer). Der Test zeigte weiters, daß weniger unechte Veränderungen erfaßt wurden, wenn das Interview mit Vorgaben durchgeführt wurde (mehr als $60 \%$ tatsächlich stattgefundenen Veränderungen gegenüber $20-40 \%$ ohne Vorgaben je nachdem, ob der interviewer dieselbe Person war oder nicht). Interviews mit Vorgaben werden lediglich in den Erhebungen in Portugal, Schweden und im Vereinigten Königreich durchgeführt.

\subsection{Feldarbeit für eine kontinuierliche Erhebung}

Eine kontinuierliche Erhebung erfordert eine andere Organisation der Feldarbeit als eine Erhebung in einer oder wenigen Wochen. Weil die Interviews über einen längeren Zeitraum verteilt werden können, werden weniger Interviewer benötigt, während ihre Aufgaben umfangreicher sein werden als die im Rahmen einer Erhebung in einer oder wenigen Wochen (Tabelle 3). Weil die Zusammenarbeit zwischen den Interviewern und dem nationalen statistischen Amt intensiver ist, lohnen sich Investitionen in Ausbildung und Überwachung. Eine bessere Ausbildung und Überwachung wird die Datenqualität verbessern, wobei eine unzureichende Ausbildung nicht durch Überwachung kompensiert werden kann.

Der Interviewer ist das entscheidende Bindeglied zwischen dem Statistiker und der Auskunftsperson. Er hat direkten Kontakt zur Auskunftsperson und verwendet den Fragebogen zur Sammlung der Daten. Der Statistiker muß den Interviewer kontrollieren, damit die Validität der Interviewdaten gewährleistet ist. Der Interviewer hat drei Hauptaufgaben: i) Kontakt zur Auskunftsperson aufnehmen und sie für eine Befragung gewinnen, ii) die Befragung durchführen und iii) die Antworten festhalten.

1 Die Arbeitsplatzbindung setzt sich aus drei Kategorien zusammen: unbefristeter Vertrag, befristeter Vertrag oder Aushilfstätigkeit im vergangenen Jahr, und ohne Beschäftigung im vergangenen Jahr. 
Tabelle 3: Interviewer, Aufwand des Interviewers, Ausbildung des Interviewers und Kontrolle der Leistung des Interviewers

\begin{tabular}{|c|c|c|c|c|c|c|}
\hline & $\begin{array}{l}\text { Anzahl } \\
\text { der } \\
\text { Inter- } \\
\text { viewer }\end{array}$ & $\begin{array}{l}\text { Durchschnitt n } \\
\text { der Interviews } \\
\text { pro Interview- } \\
\text { er (jährlich, } \\
\text { ausgenommen } \\
\text { postalische } \\
\text { Befragung) }\end{array}$ & $\begin{array}{l}\text { Interviewer: } \\
\text { Merkmale }\end{array}$ & $\begin{array}{l}\text { Ausbildung: } \\
\text { Dauer }\end{array}$ & $\begin{array}{l}\text { Ausbildungs- } \\
\text { personal }\end{array}$ & $\begin{array}{l}\text { Kontrolle der } \\
\text { Leistung des } \\
\text { Interviewer }\end{array}$ \\
\hline S & $\begin{array}{l}110 \\
\text { (CATI: } \\
20 \text { von } \\
\text { der Zen- } \\
\text { tralein- } \\
\text { heit, } 90 \\
\text { von zu- } \\
\text { hause) }\end{array}$ & 1960 & & 5 Tage & $\begin{array}{l}\text { Personal des } \\
\text { Zentralamtes }\end{array}$ & $\begin{array}{l}2 \% \text { Erhebung } \\
\text { mit erneutem } \\
\text { Interview }\end{array}$ \\
\hline Dk & 38 & 1150 & & $\begin{array}{l}4 \text { halbe } \\
\text { Tage }\end{array}$ & $\begin{array}{l}4 \text { Aufsichtsperso- } \\
\text { nen/Kodierer }\end{array}$ & $\begin{array}{l}\text { Aufsichtspers. } \\
\text { hören im Ge- } \\
\text { spräch mit }\end{array}$ \\
\hline $\mathrm{E}$ & 270 & 950 & & 2 Tage & $\begin{array}{l}1 \text { Aufsichtspers. } \\
\text { für die Erhebung } \\
\text { in jeder Provinz }\end{array}$ & $\begin{array}{l}5 \% \text { Erhebung } \\
\text { mit erneutem } \\
\text { Interview }\end{array}$ \\
\hline Fin & $\begin{array}{l}155 \\
\text { (CAT1) }\end{array}$ & 930 & & 3 Tage & $\begin{array}{l}3 \text { Mitglieder des } \\
\text { Personals }\end{array}$ & \\
\hline $\mathrm{P}$ & 112 & 800 & & $\begin{array}{l}\text { Ausbildung } \\
\text { vor Ort }\end{array}$ & $\begin{array}{l}4 \text { regionale } \\
\text { Zentren }\end{array}$ & $\begin{array}{l}5 \% \text { Erhebung } \\
\text { mit erneutem } \\
\text { Interview }\end{array}$ \\
\hline UK & 420 & 600 & & 5 Tage & $\begin{array}{l}3 \text { Mitglieder des } \\
\text { Personals }\end{array}$ & $\begin{array}{l}\text { Aufsichtspersone } \\
\mathrm{n} \text { als Begleitung } \\
\text { oder Zuhörer }\end{array}$ \\
\hline I & 2000 & 150 & $\begin{array}{l}\text { lokale } \\
\text { Beamte }\end{array}$ & & dezentralisiert & $\begin{array}{l}\text { Überprüfung } \\
\text { Erhebung (1994) }\end{array}$ \\
\hline $\mathrm{Nl}$ & 380 & 325 & & 7 Tage & $\begin{array}{l}7 \text { Mitglieder des } \\
\text { Personals }\end{array}$ & $\begin{array}{l}\text { Erhebung über } \\
\text { die Zufriedenheit } \\
\text { der Auskunfts- } \\
\text { personen }\end{array}$ \\
\hline El & 500 & 125 & $\begin{array}{l}\text { Beamte } \\
\text { (Athen) und } \\
\text { zeitweilige } \\
\text { Interviewer }\end{array}$ & $\begin{array}{l}2 \text { Tage }+ \\
\text { Ausbildung } \\
\text { vor Ort }\end{array}$ & & $\begin{array}{l}\text { Kontrolle der } \\
\text { ausgefüllten } \\
\text { Fragebogen im } \\
\text { Amt }\end{array}$ \\
\hline Irl & 430 & 115 & & 5 Tage & $\begin{array}{l}40 \text { Aufsichtspers } \\
\text { o-nen vor Ort }\end{array}$ & Kontrolle im Amt \\
\hline B & 300 & 115 & Beamte & & & \\
\hline
\end{tabular}




\begin{tabular}{|l|l|l|l|l|l|l|}
\hline & $\begin{array}{l}\text { Anzahl } \\
\text { der } \\
\text { Inter- } \\
\text { viewer }\end{array}$ & $\begin{array}{l}\text { Durchschnitt } \mathrm{n} \\
\text { der Interviews } \\
\text { pro Interview- } \\
\text { er }\end{array}$ & $\begin{array}{l}\text { Interviewer: } \\
\text { Merkmale }\end{array}$ & $\begin{array}{l}\text { Ausbildung: } \\
\text { Dauer }\end{array}$ & $\begin{array}{l}\text { Ausbildungs- } \\
\text { personal }\end{array}$ & Leistungskontr. \\
\hline A & 1200 & 101 & $\begin{array}{l}\text { lokale } \\
\text { Beamte }\end{array}$ & 1 Tag & $\begin{array}{l}\text { 1 oder 2 Mit- } \\
\text { glieder des Per- } \\
\text { sonals im Lan- } \\
\text { desamt }\end{array}$ & Kontrolle im Amt \\
\hline F & 1000 & 80 & $\begin{array}{l}\text { Unterstüt- } \\
\text { zung durch } \\
\text { zeitweilige } \\
\text { Interviewer }\end{array}$ & $4-5$ Tage & $\begin{array}{l}\text { 20 regionale } \\
\text { Zentren }\end{array}$ & Kontrolle im Amt \\
\hline D & 8000 & 40 & $\begin{array}{l}\text { lokale } \\
\text { Beamte und } \\
\text { zeitweilige } \\
\text { Interviewer }\end{array}$ & 1 Tag & $\begin{array}{l}16 \text { statistische } \\
\text { Landesämter }\end{array}$ & \\
\hline L & 170 & 30 & $\begin{array}{l}\text { lokale } \\
\text { Beamte }\end{array}$ & & & \\
\hline
\end{tabular}

Standardisierte Interviews sind die Voraussetzung dafür, daß die Bedeutung der Fragen bei allen Interviews gleich bleibt und der Absicht des Statistikers entspricht. In einem standardisierten Interview werden die Antworten nicht durch den Interviewer (Fowler and Mangione, 1990) beeinflußt. Ein standardisiertes Interview bedeutet:

- Fragen und ihre Antwortkategorien werden immer ohne Abweichung genau so gelesen, wie sie im Fragebogen festgehalten oder auf dem Bildschirm dargestellt werden,

- Der Interviewer ist auf Klärung bedacht, wenn der Befragte unvollständig oder unzureichend antwortet, ohne diesen zu beeinflussen; eine Möglichkeit zur Klärung ohne Beeinflussung des Befragten besteht in der Wiederholung der Frage, unter Umständen mit Betonung des Teils, den der Befragte anscheinend nicht verstanden hat, und mit der Frage "Wie meinen Sie das?" oder “Können Sie das ausführen?",

- der Interviewer erfaßt die Antworten objektiv,

- neutrale Kommunikation: Der Interviewer sollte den Inhalt der Antworten des Befragten nicht bewerten.

Die Durchführung standardisierter Interviews ist nicht einfach. Es ist überraschend, wieviele Interviewer ihre eigenen Fragen einsetzen. Für standardisierte Interviews ist eine intensive Ausbildung der Interviewer und eine Kontrolle ihrer Leistung notwendig. Die Ausbildung sollte Übungen und überwachte praktische Anwendungen zusätzlich zu Vorlesungen und Demonstrationen beinhalten.

Eine eintägige Ausbildung ist eindeutig unzureichend, um die Technik standardisierter Interviews zusätzlich zu den Verwaltungsverfahren, der ersten Vorstellung der Erhebung an der Tür oder am Telefon und der Struktur des Fragebogens zu erlernen. 
Nach einer eintägigen Ausbildung entwickeln Interviewer später gerne ihre eigenen Techniken. Eine Ausbildung von mindestens 2 oder 3 Tagen erscheint notwendig. Erfolgt diese Ausbildung durch eine kleine Gruppe von Ausbildern des Zentralamtes (z.B. Schweden, Vereinigtes Königreich), trägt dies mit Sicherheit zur Vereinheitlichung von Interviewtechniken bei. In Italien hingegen ist die Organisation der Feldarbeit stark dezentralisiert und beeinträchtigt so möglicherweise die Einheitlichkeit der Datenerhebung. Die Interviewer werden von den Kommunen eingestellt und ausgebildet. Die Kommunen werden nicht direkt vom statistischen Zentralamt in Rom überwacht, sondern von regionalen Büros und den Handelskammern. Wenn die Erhebung innerhalb einer oder mehrerer Wochen durchgeführt wird, werden lokale Beamte oder andere zeitweilige Interviewer für die Arbeitskräfteerhebung herangezogen, oder aber sie unterstützen die regulären Interviewer (z.B. Frankreich, Deutschland und Österreich).

Zusätzlich zur Ausbildung ist eine Kontrolle der Leistung der Interviewer notwendig. Diese Kontrolle kann die Überwachung der Interviews beinhalten, ein erneutes Interview oder eine Kontrolle der ausgefüllten Fragebogen im Amt. Eine erste Möglichkeit zur Überwachung von Interviews besteht in der Aufnahme persönlicher Interviews auf Band, in der Begleitung der Interviewer vor Ort oder im Zuhören bei telefonischen Befragungen (Vereinigtes Königreich, Dänemark). Eine weitere Möglichkeit besteht im erneuten Interview einer Unterstichprobe mit demselben Fragebogen eine Woche später - doch erneut zum Erwerbsstatus in der Bezugswoche des ersten Interviews. Dieselben Fragen werden derselben Person gestellt, und die Interviews können von Aufsichtspersonen durchgeführt werden (Spanien, Portugal). Unstimmigkeiten können Hinweise auf eine schlechte Leistung des Interviewers sein. Die Kontrolle ausgefüllter Fragebogen im Amt ist eine weniger effiziente Methode. Neben einer Kontrolle von Vollständigkeit und verwendeten Filtern wird die Adäquatheit der gelieferten Informationen überprüft, in erster Linie in Bezug auf die Variablen wie beispielsweise Beruf und Wirtschaftszweig, die kodiert werden müssen. Bei rechnergestützten Befragungen sind die meisten dieser Kontrollen redundant. Sie sind kaum geeignet als Kontrolle für das Interviewverfahren. Eine Aufsichtsperson kann auf der Grundlage dieser Überprüfung nicht erkennen, ob eine Frage genau so gestellt wurde, wie sie vorformuliert ist, oder ob der Interviewer eine Klärung ohne Beeinflussung des Befragen herbeigeführt hat.

\subsection{Praktische Überlegungen}

Eine kontinuierliche Datensammlung und -aufbereitung kann besser verwaltet werden. Ein stetiger Strom von Interviews und Interviewdaten bietet den Vorteil, daß die erforderlichen Humanressourcen und maschinelle Unterstützung besser geplant werden können als wenn nur zu bestimmten Zeitpunkten bestimmte Mittel benötigt werden (z.B. die Verteilung der Stichprobenadressen zwei Wochen vor Beginn der Interviews und Dateneingang nach der Beendigung der Feldarbeit). Der Zeitraum bis zur Veröffentlichung der ersten Ergebnisse scheint ebenfalls kürzer bei einer kontinuierlichen Erhebung (z.B. 12 Tage nach dem letzten Interview in Schweden, 6 Wochen nach dem letzten Interview in Spanien und im Vereinigten Königreich). 
Die Einführung einer kontinuierlichen Datenerhebung und -aufbereitung muß nicht auf die Arbeitskräfteerhebung begrenzt sein. Wenn eine größere Zahl von Haushaltserhebungen betroffen ist, liegt der Bedarf an einer kontinuierlichen Datensammlung und Datenverarbeitung auf der Hand (ein Problem bei einer Erhebung im Rahmen eines nichtkontinuierlichen Erhebungsverfahrens führt $\mathrm{zu}$ einer Verzögerung für viele nachfolgende Erhebungen). Die Neuorganisation einer großen Stichprobenerhebung beispielsweise der Arbeitskräfteerhebung - und der Übergang zu einer kontinuierlichen Erhebung kann zu einem koordinierten Prozeß bei der Neugestaltung der Designs der Haushaltserhebungen führen.

\section{Gewichtungsverfahren}

Die Arbeitskräfteerhebung muß für die Bevölkerung repräsentativ sein. Dies bedeutet, daß die Verteilung nach Alter, Geschlecht und Region nach Maßgabe der Arbeitskräfteerhebung den Daten aus dem Bevölkerungsregister oder Extrapolationen aus der Volkszählung entsprechen. Ein Vergleich der Daten aus der Arbeitskräfteerhebung für 1992-94 nach Alter und Geschlecht mit Bevölkerungsgesamtwerten zeigte, daß die Übereinstimmung je nach Mitgliedstaat unterschiedlich ist. Weil ein Zusammenhang besteht zwischen den demographischen Variablen und dem Erwerbsstatus, könnte diese Variable verzerrt werden. Abhängig von der Verfügbarkeit zuverlässiger Bevölkerungs-Benchmarks auf regionaler Ebene sollte eine Ex-postStratifizierung nach Geschlecht, Alter (Fünfjahresaltersgruppen) und Region vorgenommen werden.

Zweck der Gewichtung der Stichprobendaten ist es, zu repräsentativen Daten zu gelangen. Abweichungen zwischen den (ungewichteten) Stichprobenergebnissen und der Grundgesamtheit werden aus folgenden Gründen gewichtet:

- Bei einer Gewichtung werden unterschiedliche Stichprobenwahrscheinlichkeiten berücksichtigt, z.B. Überrepräsentation der registrierten Arbeitslosen, regionale Überrepräsentation oder ungleiche Stichprobenwahrscheinlichkeit von Haushalten, die aus einem Bevölkerungsregister ausgewählt wurden.

- Durch eine Gewichtung wird die durch Non-Response bedingte Verzerrung verringert. Eine nachträgliche Schichtung ist effizient, wenn eine Hilfs- oder Stratifizierungsvariable (z.B. Alter) in Beziehung steht zum Antwort-Indikator und zur Zielvariable (z.B. Erwerbsstatus). Ferner liegt innerhalb der Schichten eine bessere Übereinstimmung zwischen der beobachteten Verteilung des Erwerbsstatus und der Verteilung in der Grundgesamtheit vor als vor der Ex-post-Stratifizierung. Die Definition dieser Schichten ist abhängig von der Verfügbarkeit von Daten sowohl über die Auskunftgebenden als auch über die Erhebungsausfälle. Beispiele für Schichtungsvariablen sind die Art der Wohnung oder der Wohnort (Frankreich) und die Nationalität (Deutschland und Österreich, Tabelle 4).

- Im Falle eines Bevölkerungsregisters stehen mehrere andere nützliche Variablen zur Verfügung (Schweden, Dänemark). 
Tabelle 4: Ex-post-Stratifizierung

\begin{tabular}{|c|c|c|c|c|c|c|}
\hline & Region & Geschlecht & Alter & $\begin{array}{l}\text { Familienstand } \\
\text { oder Haus- } \\
\text { haltsgröße }\end{array}$ & $\begin{array}{l}\text { Staatsange- } \\
\text { hörigkeit (und } \\
\text { Geburtsland) }\end{array}$ & Sonstiges \\
\hline El & \multicolumn{6}{|c|}{ Horvitz-Thompson-Schätzer } \\
\hline $\mathrm{P}$ & \multicolumn{6}{|c|}{ Horvitz-Thompson-Schätzer, geschichtet nach Region } \\
\hline $\mathrm{E}$ & * & & $*(<16,>=16$ & & & \\
\hline $\mathrm{L}$ & & $*$ & $*$ & & & \\
\hline Irl & $*$ & $*$ & * & & & \\
\hline B & * & $*$ & $\begin{array}{l}* \quad \text { (5 Jahres- } \\
\text { gruppe) }\end{array}$ & & & \\
\hline $\mathrm{I}$ & $*$ & $*$ & $*$ ( 5 Klassen $)$ & & & \\
\hline Fin & $*$ & $*$ & $*$ & & & \\
\hline A & * & $*$ & $\begin{array}{l}* \quad \text { (5 Jahres- } \\
\text { gruppe) }\end{array}$ & & $*$ & \\
\hline $\mathrm{D}$ & $*$ & $*$ & $*(<60,>=60)$ & * (HHgröße) & & \\
\hline $\mathrm{F}$ & & $*$ & $\begin{array}{l}* \quad \text { (5 Jahres- } \\
\text { gruppe) }\end{array}$ & & & $\begin{array}{l}\text { Stadt/Land/Pa } \\
\text { ris, Rotations- } \\
\text { gruppe, } \\
\text { Einfamilien- } \\
\text { /N+familien- } \\
\text { haus }\end{array}$ \\
\hline Dk & & $*$ & * (3 Klassen) & & & $\begin{array}{l}\text { Erwerbsstatus } \\
\text { nach Register, } \\
\text { Einkommen, } \\
\text { Bildung und/ } \\
\text { oder Wirt- } \\
\text { schaftszweig }\end{array}$ \\
\hline $\mathrm{S}$ & & $*$ & $\begin{array}{l}*(5-\text { oder } 10- \\
\text { Jahres- } \\
\text { gruppen })\end{array}$ & & & $\begin{array}{l}\text { erwerbstätig/- } \\
\text { registriert } \\
\text { erwerbslos, } \\
\text { Wirtschafts- } \\
\text { zweig sofern } \\
\text { erwerbstätig }\end{array}$ \\
\hline VK & $*$ & $*$ & $\begin{array}{l}* \text { (einzelne } \\
\text { Jahre bei den } \\
\text { 16-24-jährig- } \\
\text { en und } 5 \text { Jahr- } \\
\text { esgruppe) }\end{array}$ & & & \\
\hline $\mathrm{Nl}$ & $*$ & $*$ & $*$ & $\begin{array}{l}* \quad \text { (Familien- } \\
\text { stand) }\end{array}$ & $*$ & \\
\hline
\end{tabular}


- Durch eine Gewichtung wird die Diskrepanz zwischen Randverteilungen in der Stichprobe und in der Grundgesamtheit verringert. Die Ex-post-Stratifizierung ist abhängig von der Verfügbarkeit zuverlässiger Benchmarks der Grundgesamtheit. Quellen sind Registerzählungen, Extrapolationen der Volkszählung oder Benchmarkverteilungen aus sehr großen Stichproben (z.B. Unternehmenserhebungen). Die üblichen Schichtungsvariablen sind Region, Geschlecht und Alter. Die üblichen Schichtungsvariablen sind Region, Geschlecht und Alter. Die Klassifikation nach Alter ist unterschiedlich: Zwischen einer Bandbreite von 10-15 Jahren (Italien) und einer detaillierteren Bandbreite von 5 Jahren (Belgien). Aufgrund der Bedeutung der Schätzungen der Jugendarbeitslosigkeit werden im Vereinigten Königreich für Personen unter 26 Jahren Ein-Jahresklassen zugrundegelegt.

- Diese drei Gründe rechtfertigen die Ex-post-Stratifizierung als eine Methode zur erneuten Gewichtung der Stichprobendaten nach der Auswahl. Ein letzter Grund steht in Zusammenhang mit der Varianz der Schätzungen: Durch Ex-postStratifizierung wird die Varianz im Vergleich zur stichprobendesignbedingten Varianz in der Regel verringert.

Bei der Gewichtung für ungleiche Stichprobenwahrscheinlichkeiten, Non-ResponseAnpassung und Verteilungen der Grundgesamtheit handelt es sich um unterschiedliche Stufen im Gewichtungsverfahren. Es muß nicht in jeder Stufe eine vollständige Kreuzklassifizierung für alle Schichtungsvariablen vorgenommen werden. Lineare Regressionsgewichtung und iterative proportionale Anpassung ermöglichen gesonderte Klassifikationen. Die Mindestforderung einer Ex-post-Stratifizierung nach Geschlecht, Alter und Region schließt eine Ex-post-Stratifizierung nach anderen Variablen nicht aus, wenn dies für nationale Zwecke erforderlich ist.

Die Arbeitskräfteerhebung ist nicht nur eine Quelle für Individualdaten, sondern auch für Haushaltsdaten (Ziel iv). Um Konsistenz zwischen Haushaltsschätzungen und individuenbasierten Schätzungen zu erreichen, müssen gleiche Gewichte für alle Haushaltsmitglieder verwendet werden. Ein und dasselbe Gewicht für alle Haushaltsmitglieder bietet für die Benutzer der Erhebungsdaten einen offensichtlichen Vorteil.

Durch diese Randbedingung beim Gewichtungsverfahren wird die spätere Berechnung und Auswahl eines speziellen Gewichts für Haushaltsdaten vermieden (z.B. das Gewicht der Referenzperson oder ein Durchschnitt der Gewichte aller Haushaltsmitglieder ${ }^{2}$ ). Schätzungen zum Erwerbsstatus auf der Grundlage eines einzigen Gewichts innerhalb eines Haushalts und ihrer Standardfehler ergeben praktisch dasselbe wie auf der Grundlage individueller Gewichte (Andersson, 1997). In Frankreich und in den Niederlanden werden die Gewichte unter der Nebenbedingung gleicher Gewichte für alle Haushaltsmitglieder festgelegt.

2 Im Mikrozensus in Österreich sind die Gewichte der Haushaltsmitglieder Durchschnittswerte; Konvergenz der individuellen Gewichte und des Durchschnittsgewichts wird durch ein iteratives Verfahren erreicht. 


\section{Variablen}

\subsection{Liste von Variablen}

Um den Aufwand für die Auskunftspersonen und die Interviewkosten zu verringern, sind die $\mathrm{zu}$ erhebenden Daten auf einen Kernsatz von Variablen begrenzt. Eine möglichst geringe Belastung der Auskunftsperson ist wichtig, weil ihre Mitarbeit mehrfach benötigt wird (Erhebungstyp "rotierendes Panel"). Der Kernsatz der Variablen setzt sich wie folgt zusammen:

- Variablen zum demographischen Hintergrund (Geschlecht, Alter, Familienstand, familiäre Beziehungen innerhalb des Haushalts, Staatsangehörigkeit ...);

- Erwerbsstatus (erwerbstätig, auf Arbeitsuche, Verfügbarkeit);

- Merkmale der Haupttätigkeit (Wirtschaftszweig, Beruf, Erwerbsstatus, Jahr und Monat, in dem die Person ihre Arbeit aufnahm, Anzahl der in der Regel gearbeiteten Stunden ...);

- Merkmale einer zweiten Tätigkeit;

- sichtbare Unterbeschäftigung (die Fragen danach, ob man mehr Stunden arbeiten möchte, und zur Anzahl der gewünschten Stunden sind fakultativ). Die Unterscheidung zwischen Erwerbstätigen und Erwerbslosen gibt nur ein unvollständiges Bild des Arbeitsmarkts wieder. Viele Erwerbstätige gehen einer Teilzeitbeschäftigung nach, die möglicherweise lediglich einige Stunden in der Woche umfaßt. Die sichtbare Unterbeschäftigung ist ein sinnvoller ergänzender Indikator. Sie erfaßt die Personen, die zusätzliche Stunden arbeiten möchten (an einem anderen Arbeitsplatz oder an ihrem jetzigen Arbeitsplatz), die nach einem anderen Platz suchen oder ihre jetzige Beschäftigung ändern möchten und dem Arbeitsmarkt zur Verfügung stehen;

- bisherige Berufserfahrung erwerbsloser Personen;

- abgeschlossene Ausbildung und Beteiligung an Ausbildungs- oder Fortbildungsmaßnahmen in den letzten vier Wochen;

- Haupterwerbsstatus ein Jahr vor der Erhebung;

- Löhne und Gehälter (Nettoverdienst und zusätzliche Zahlungen, die nicht monatlich erfolgen), Arbeitslosengeld, Zahlungen bei Krankheit und Erwerbsunfähigkeit); diese Angaben sind fakultativ.

\subsection{Gesamtzahl der arbeitenden Personen und Zahl der gear- beiteten Stunden}

Eine kontinuierliche Erhebung liefert eher valide Schätzungen über die Anzahl der arbeitenden Personen und über die Anzahl der gearbeiteten Stunden als eine Erhebung mit einem festgelegten Berichtszeitraum. Abweichungen der Schätzwerte auf der 
Grundlage einer einzigen Berichtswoche in einem Quartal und auf der Grundlage einer gleichmäßigen Verteilung der Wochen innerhalb des Quartals sind u.a. auf die Berücksichtigung/den Ausschluß von Feiertagen und das konstante/variable Intervall zwischen zwei festen Referenzwochen zurückzuführen.

Eine festgelegte Berichtswoche ist in der Regel eine Woche ohne Feiertage. Eine solche typische Woche wird ausgewählt, um Störeffekte durch besondere Ereignisse auf die Schätzungen zu vermeiden. Die Erwerbstätigen sind am Arbeitsplatz anwesend, und es kommt nicht zu einem plötzlichen Anstieg von Gelegenheitstätigkeiten in bestimmten Wirtschaftszweigen. Da in einer typischen Woche besondere Ereignisse ausgeschlossen werden, sind diese hochgerechneten Daten keine zuverlässigen Schätzwerte der Gesamtzahl der arbeitenden Personen in einem bestimmten Quartal oder Jahr. Ferner wird in einer bestimmten Berichtswoche nicht die Abwesenheit vom Arbeitsplatz auf Grund von Feiertagen oder Jahresurlaub gemessen. Daher kann die Gesamtzahl der gearbeiteten Stunden (das Arbeitsvolumen) nicht aus einer Erhebung mit einer festgelegten Berichtswoche abgeleitet werden. Außerdem werden in einer festgelegten Berichtswoche nicht der Anstieg oder Rückgang der Beschäftigung in den anderen Wochen eines Quartals festgehalten. Das geschätzte Beschäftigungsniveau wird unter Umständen auf der Grundlage von Daten aus einer einzigen Berichtswoche nicht nur verzerrt, sondern der tatsächliche Trend zwischen zwei Quartalen kann sogar im Gegensatz stehen zum beobachteten Trend auf der Grundlage der Daten aus einer einzigen Berichtswoche. Schließlich kann sich das Intervall zwischen den Berichtswochen in zwei Quartalen ändern, wenn die Verschiebung einer Berichtswoche in einem neuen Jahr notwendig ist, um zu vermeiden, daß die Berichtswoche einen Feiertag beinhaltet (Wallgren und Wallgren, 1997). Die Interpretation der Veränderungs-schätzungen ist schwierig, wenn diese sich auf unterschiedliche Abstände zwischen den Berichtswochen beziehen (der Anstieg - oder Rückgang - der Erwerbstätigen könnte teilweise auf eine Verschiebung der Berichtswoche zurückzuführen sein). Außerdem könnte eine Verschiebung der Berichtswoche wegen des saisonalen Musters innerhalb eines Quartals zu einen Bruch in der Reihe vierteljährlicher Beschäftigungschätzungen führen. Der Trend auf der Grundlage einer Verschiebung in der Berichtswoche könnte von dem wahren Trend auf der Grundlage eines konstanten Intervalls abweichen.

Die Schätzwerte auf der Grundlage einer kontinuierlichen Erhebung sind Durchschnittswerte, z.B. der Durchschnitt der Anzahl erwerbstätiger Personen in einem Quartal. Der Berichtszeitraum besteht aus allen Wochen in einem Quartal. Besondere Ereignisse und Gelegenheitsarbeit werden berücksichtigt. Die hochgerechneten Gesamtwerte sind valide Schätzwerte der Gesamtzahl der arbeitenden Personen und der Gesamtzahl der gearbeiteten Stunden.

Die Berichtsperiode des Mikrozensus in Österreich ist nicht auf eine einzige Woche begrenzt, sondern umfaßt die ersten drei (vollen) Wochen im letzten Monat eines Quartals (März, Juni, September und Dezember). Dadurch, daß die Berichtswochen festgelegt sind, wird das Problem eines variablen Intervalls zwischen den Berichtszeiträumen in zwei Quartalen vermieden. Es können jedoch keine gültigen Schätzwerte zur Anzahl der arbeitenden Personen geliefert werden, weil es sich bei der Erhebung nicht um eine Zufallsstichprobe der Grundgesamtheit der arbeitenden Personen in einem Quartal handelt. Entsprechend kann ebenfalls keine valide Volumenschätzung zur 
Anzahl der gearbeiteten Stunden geliefert werden, weil die Anzahl der Stunden, in denen Abwesenheit aufgrund von Feiertagen oder Jahresurlaub vorlag, nicht erfaßt werden.

\subsection{Messung von Beschäftigung und Arbeitslosigkeit}

Bisher war die einzige Grundlage für die internationale Vergleichbarkeit der Daten über Beschäftigung und Arbeitslosigkeit die Verwendung derselben Definition (13. Internationale Konferenz der Arbeitsstatistiker, 1982). Angesichts der politischen und wirtschaftlichen Bedeutung der Erhebungsergebnisse auf EU-Ebene ist jedoch eine Verbesserung der internationalen Vergleichbarkeit notwendig. Dies kann durch eine konvergente Messung von Beschäftigung und Arbeitslosigkeit erreicht werden. Angesichts der Unterschiede zwischen den nationalen Fragebogen ist die Vergleichbarkeit der Schätzungen anzuzweifeln. Deshalb entwickelte Eurostat 12 Grundsätze für das Design der Fragen zum Erwerbsstatus (Arbeitsgruppe Beschäftigungsstatistik im Oktober 1996). Diese Grundsätze ${ }^{3}$ spezifizieren die internationale Definition. Die Auswirkungen der Grundsätze auf die Schätzungen werden derzeit von den nationalen statistischen Ämtern untersucht.

\section{Ergänzungen zur Arbeitskräfteerhebung: Ad-hoc-Module}

Ein kleiner festgelegter Satz von Grundvariablen bietet die Möglichkeit, Ergänzungen zur Arbeitskräfteerhebung vorzunehmen, ohne die Belastung für die Auskunftsperson zu erhöhen. Vorteile einer Ergänzungserhebung zur Arbeitskräfteerhebung liegen darin, daß die Daten zum Erwerbsstatus aus der Arbeitskräfteerhebung für multivariate Analysen verfügbar sind und daß die Auswahl einer Stichprobe auf der Grundlage dieser Variablen möglich ist. Diese Ergänzungen zur Arbeitskräfteerhebung gibt es in Österreich, Schweden und Frankreich. In Österreich und in Schweden werden die Ergänzungen in jedem Quartal geändert. Beispiele von Ergänzungen in Österreich sind Wohnung und Unterkunft, Zeitverwendung, Arbeitsbedingungen. Beispiele von Ergänzungen in Schweden sind die jährliche Erhebung $\mathrm{zu}$ den Arbeitsformen (retrospektive Fragen) sowie die Arbeitsbedingungen und Arbeitsumgebung.

Eurostat wird in Zusammenarbeit mit den Mitgliedstaaten ein Mehrjahresprogramm mit Ad-hoc-Modulen erstellen. Diese Ergänzungen liefern unabdingbare Informationen über den Arbeitsmarkt; sie sind wesentlicher Teil der Arbeitskräfteerhebung als Hauptquelle für Daten zur Erwerbsbevölkerung.

3 Die 12 Grundsätze beziehen sich auf i) den Ausschluß von Kontexteffekten und Filtern, ii) die Aufnahme von Stichwörtern für spezielle Kategorien erwerbstätiger Personen und Personen, die bereits einen Arbeitsplatz gefunden haben, iii) die Berichtszeiträume und iv) die Unterscheidung zwischen aktiven und passiven Methoden zur Arbeitsplatzsuche. 


\section{Durchführung der Erhebung}

Die Mitgliedstaaten sind für die Durchführung der Erhebung verantwortlich. Unter Berücksichtigung der internationalen Vergleichbarkeit bestimmen die Mitgliedstaaten das geeignete Stichprobendesign und die geeigneten Schätzer, sie führen die Feldarbeit und die Datenaufbereitung durch und geben die Daten auf nationaler Ebene frei. Anschließend liefern sie die Individualdatensätze an Eurostat im festgelegten Format. Dieses Format spezifiziert die Variablen, ihre Werte oder Antwort-Kategorien, ihre Filter und ihre logischen Beziehungen zu anderen Variablen. Neben diesen technischen Angaben verringern eine Definition und eine detailliertere Erläuterung der Variablen die Gefahr von Mehrfachinterpretationen und liefern die Grundlage für vergleichbare Daten auf EU-Ebene.

\section{Frist für die Übermittlung der Ergebnisse}

Angesichts des Ziels der Arbeitskräfteerhebung, kurzfristige Indikatoren zu liefern, ist Aktualität von wesentlicher Bedeutung. Eine allgemeine Anforderung ist, daß die Daten innerhalb eines Zeitraums zur Verfügung stehen, der nicht länger ist als der Zeitraum, der beschrieben wird. Innerhalb von zwölf Wochen nach Ablauf des Berichtsquartals übermitteln die Mitgliedstaaten die Erhebungsergebnisse an Eurostat.

\section{Abschließende Bemerkungen von Dr. A. Franz, ÖStat}

Aloïs Van Bastelaer (EUROSTAT) wurde von der Österreichischen Statistischen Gesellschaft eingeladen, vor der Gesellschaft zu diesem Gesamtkomplex einen Vortrag zu halten, der folgende Themen ansprach:

Hintergrund der neuen Verordnung; Ziele der "kontinuierlichen" AKE; zu erfassende Gesamtheiten; Erhebungseinheiten; Anforderungen an die statistische Genauigkeit; Häufigkeit der Erhebung und Bezugszeitraum; Gewichtungsverfahren; Variablen; Ergänzungen zur AKE (ad-hoc-Module); Übermittlungsfristen.

Der gute Besuch und die rege Diskussion dieser Veranstaltung zeigte, daß die Hochrangigkeit des Themas wohl verstanden wird. Dabei ist die AKE als statistisches Instrument in der Literatur relativ wenig behandelt. Deshalb wurde es für nützlich befunden, das Manuskript in der Zeitschrift für Statistik zu veröffentlichen und so einem weiteren Personenkreis zugänglich zu machen. Es handelt sich bei diesem Beitrag also nicht um einen wissenschaftlichen Aufsatz wie gewohnt (d.h. primär für diese Zeitschrift verfaßt), und es weicht die Darstellungsweise in manchen Punkten ab. 


\section{Literatur}

C. Andersson. Continuous labour force surveys: performance analysis of a single weight procedure. $S C B$, internal report, 1997.

F.J. Fowler and T.W. Mangione. Standardized survey interviewing. Sage publications, Newbury park, 1990.

D.H. Hill. The relative empirical validity of dependent and independent data collection in a panel survey. Journal of Official Statistics 10, 1994, p. 359 - 380.

Statistics Sweden. Revision of contents and definitions in the Swedish Labour force surveys (Revision von Inhalt und Definitionen in den schwedischen Arbeitskräfteerhebungen). SCB, Örebro, 1989.

B. Wallgren und A. Wallgren. Verknüpfung eines Systems von Zeitreihen: Umstellung der Arbeitskräfteerhebungen 1987-1992 (in schwedisch). SCB, Örebro, 1997.

Adresse des Autors:

Dr. Aloïs Van Bastelaer

Bâtiment Jean Monnet

L-2920 Luxemburg-Kirchberg

Luxemburg

E-mail: ALOIS.VANBASTELAER@EUROSTAT.CEC.BE 